\title{
Exosomes derived from bone marrow stromal cells decrease the sensitivity of leukemic cells to etoposide
}

\author{
JIANLING WANG ${ }^{1,2}$, DONG LI ${ }^{3}$, YONG ZHUANG ${ }^{2}$, JINQIU FU $^{2}, \mathrm{XUE} \mathrm{LI}^{2}, \mathrm{QING} \mathrm{SHI}^{3}$ and XIULI JU ${ }^{1,2}$ \\ ${ }^{1}$ Shenzhen Research Institute of Shandong University, Shenzhen, Guangdong 518057; ${ }^{2}$ Department of Pediatrics and \\ ${ }^{3}$ Cyromedicine Lab of Qilu Hospital, Qilu Hospital, Shandong University, Jinan, Shandong 250012, P.R. China
}

Received March 5, 2016; Accepted December 1, 2016

DOI: $10.3892 / 01.2017 .6509$

\begin{abstract}
The aim of the study was to investigate the effect of exosomes derived from bone marrow stromal cells (BM-SCs) on the chemoresistant characteristics of nalm- 6 cells treated with etoposide (VP16). The present study isolated exosomes from BM-SC-conditioned medium by using standard differential centrifugation steps and detected the expression of 70 kilodalton heat shock proteins (HSP70) and lysosomal-associated membrane protein 3 (CD63) in exosomes by western blot analysis. Nalm- 6 cells were co-cultured with exosomes in the presence of VP16. Cell viability and apoptosis were then detected using the Cell Counting Kit-8 method and Annexin-V/propidium iodide, respectively. Finally, protein levels of B-cell lymphoma 2 (BCL-2), BCL-2-like protein 4 (BAX), caspase-3, and poly ADP-ribose polymerase (PARP) were examined by western blot analysis. Exosomes were successfully isolated from the conditioned medium and confirmed by the expression of HSP70 and CD63. BM-SC-derived exosomes increased the viability of nalm- 6 cells in the presence of VP16 and inhibited the apoptosis induced by VP16. Western blot analysis results showed that exosomes can block the significant reduction of BCL-2, full-length caspase-3 and full-length PARP, while preventing the increase of BAX, cleaved caspase- 3 and cleaved PARP induced by VP16. Exosomes derived from BM-SCs can protect nalm-6 cells from VP16-induced apoptosis to maintain their survival and induce resistance to VP16. In addition, BCL-2/BAX, caspase-3, and PARP may be involved in the mechanism of exosome-induced drug resistance.
\end{abstract}

\section{Introduction}

Acute lymphoblastic leukemia (ALL) is a malignant disease of the hematopoietic system that arises in the bone marrow

Correspondence to: Mrs. Xiuli Ju, Shenzhen Research Institute of Shandong University, 3688 Nanhai Avenue, Shenzhen, Guangdong 518057, P.R. China

E-mail: jxlqlyy@163.com

Key words: exosomes, chemoresistance, leukemic cells, VP16
(BM) (1). ALL is the most common type of cancer among children, but is also one of the most treatable. The majority of children with ALL either receive successful therapy or have positive outcomes, however children may relapse with poor outcomes subsequent to systemic treatments (2). These outcomes may be attributed to drug resistance of leukemia cells to chemotherapeutic reagents. Leukemia cells depend on the BM microenvironment for their growth and survival by interacting with BM-SCs.

BM-SCs are important components of the BM hematopoietic microenvironment because of their ability to self-renew and differentiate into a variety of mesodermal lineages, such as osteoblasts, chondrocytes and adipocytes (3). BM-SCs support the hematopoietic process, survival, differentiation and proliferation of hematopoietic cells, in vivo by secreting soluble cytokines, growth factors and small molecular mediators (4). Previous studies showed that BM-SCs play an important role in the initiation, development, progression and drug resistance of leukemia (5). In addition, BM-SCs affect the chemoresistance of leukemia cells during chemotherapy. Mudry et al (6) found that BM-SCs regulated the reaction of leukemia cells to chemotherapeutic drugs by protecting them against cell apoptosis induced by chemotherapeutic regimens. Although previous studies reported possible mechanisms by which BM-SCs protect leukemia cells against chemotherapeutic regimens, little is known about the exact mechanisms of chemoresistance of leukemia cells.

A series of cytokines secreted by BM-SCs can be involved in the leukemia chemoresistance process $(7,8)$. Aside from soluble factors, exosomes are an emerging tool for mediating cell-cell communications and contain enriched proteins, mRNA, and miRNAs. Exosomes are 40-100 nm-diameter nanovesicles and are secreted by various cell types upon fusion of multivesicular endosomes with plasma membranes. Exosomes mediate intercellular communications through transferring bioactive factors to other cells $(9,10)$. BM-SC-derived exosomes have been reported as a new mechanism for the paracrine action of BM-SCs (11). However, little is known about the role of BM-SC exosomes in the chemoresistance of leukemia cells.

In the present study, exosomes were isolated from BM-SCs culture medium by serial centrifugation and the effects of co-cultured BM-SCs-exosomes on the cell viability, apoptosis, and drug resistance of leukemia cells in vitro were 
investigated. The possible mechanism involving exosomes was also explored to provide additional insight into the biological role of BM-SCs-exosomes and their potential clinical applications. The present study found that BM-SCs-derived exosomes protected leukemia cells against chemotherapeutic drugs by decreasing the sensitivity of leukemia cells to chemotherapy. The current findings provide new evidence about the mechanism by which leukemia cells produce chemotherapeutic resistance.

\section{Materials and methods}

Cell culture. The human B-cell ALL nalm-6 cell line was cultured in suspension with RPMI-1640 medium (Hyclone; GE Healthcare Life Sciences, Logan, UT, USA) that contained $10 \%$ fetal bovine serum (FBS) (Gibco; Thermo Fisher Scientific, Inc., Waltham, MA, USA) and 1\% penicillin/streptomycin (Gibco; Thermo Fisher Scientific, Inc.). The cells were incubated at $37^{\circ} \mathrm{C}$ in a humidified atmosphere of $5 \%$ $\mathrm{CO}_{2}$ and passaged every 3 days to maintain a cell density of $\leq 1 \times 10^{6}$ cells $/ \mathrm{ml}$. The BM-SCs HS-5 cell line (American Type Culture Collection, Manassas, VA, USA) was cultured in high glucose Dulbecco's modified Eagle's medium (Hyclone; GE Healthcare Life Sciences) with $10 \%$ FBS and $1 \%$ penicillin/streptomycin at $37^{\circ} \mathrm{C}$ with $5 \% \mathrm{CO}_{2}$. The cells were then trypsinized and passaged at $1 \times 10^{4}$ cells $/ \mathrm{cm}^{2}$ in the medium as aforementioned. The cells were used subsequent to between 4 and 7 passages.

Isolation and identification of exosomes. BM-SCs were grown to $50 \%$ confluence in a $225-\mathrm{cm}^{2}$ flask (Corning Incorporated, Corning, NY, USA), and the culture medium was removed. The cells were washed with PBS twice and cultured in UltraCULTURE Serum-free medium (Lonza Group Ltd., Basel, Switzerland) for 48 h. Subsequently, exosomes were collected from the BM-SCs cultures following $48 \mathrm{~h}$ through standard differential centrifugation steps using a LYNX 6000 centrifuge (Thermo Fisher Scientific, Inc.). The supernatants were centrifuged at $300 \mathrm{xg}$ for $10 \mathrm{~min}$ and 2,000 $\mathrm{x}$ g for $10 \mathrm{~min}$ to remove residual cells and debris, then $10,000 \mathrm{x} g$ for $30 \mathrm{~min}$ to remove microparticles, and $100,000 \mathrm{x} g$ for $12 \mathrm{~h}$ to obtain the exosome pellets. Finally, 70 kilodalton heat shock protein (HSP70; dilution, 1:2,000; catalog no., EXOAB-Hsp70A-1; System Biosciences, Inc., Palo Alto, CA, USA) and lysosomal-associated membrane protein 3 (CD63; dilution, 1:1,000; catalog no., ab134045; Abcam, Cambridge, MA, USA) were determined by western blot analysis for the identification of exosomes. The aliquots were stored at $-80^{\circ} \mathrm{C}$ for later use, and the exosomes isolated from $5 \mathrm{ml}$ of the conditioned medium were used as a unit.

Transmission electron microscopy. Purified exosomes were fixed with $3 \%$ glutaraldehyde for $>4 \mathrm{~h}$ at $4^{\circ} \mathrm{C}$. Subsequently, the samples were washed with PBS 3 times and post-fixed with $1 \%$ osmic acid for $2 \mathrm{~h}$ at room temperature. Subsequent to a series of ethanol and acetone dehydration, penetration, embedding, and polymerization, the samples were cut into slices (thickness, $70 \mathrm{~nm}$ ) and stained with uranyl acetate and citric acid. Samples were dried and visualized under a JEOL-1200EX transmission electron microscope (JEOL,
Ltd., Tokyo, Japan) and recorded using MORADA-2 software (Olympus Corporation, Tokyo, Japan).

Cell viability assay. Chemoresistant characteristics were detected by the Cell Counting Kit- 8 assay (Beyotime Institute of Biotechnology, Shanghai, China) according to the manufacturer's protocol. Nalm- 6 cells were seeded at $4 \times 10^{4}$ cells per well in 96-well plates and co-cultured with or without $1 \mathrm{U} / \mathrm{ml}$ exosomes in the presence or absence of $0.3 \mu \mathrm{g} / \mathrm{ml}$ etoposide (VP16). A group without cells served as blank. Cell viability was examined subsequent to treatment with exosomes for $48 \mathrm{~h}$. Optical density was determined at $450 \mathrm{~nm}$ using a microplate reader. Each group was completed in triplicate, and the experiment was repeated 3 times to ensure accuracy.

Cell apoptosis analysis. Nalm-6 cells were seeded at 4x10 $/ \mathrm{ml}$ per well in serum-free medium in 12-well plates and were treated with or without $1 \mathrm{U} / \mathrm{ml} \mathrm{BM-SCs-exosomes} \mathrm{in} \mathrm{the}$ presence of $0.3 \mu \mathrm{g} / \mathrm{ml} \mathrm{VP16}$ for $48 \mathrm{~h}$. A group with nalm-6 cells alone served as the control. Collected cells were washed three times with PBS, resuspended in $100 \mu \mathrm{l}$ of binding buffer, and incubated with $5 \mu \mathrm{l}$ Annexin $\mathrm{V}$ and $10 \mu \mathrm{l}$ propidium iodide (Beyotime Institute of Biotechnology) for $15 \mathrm{~min}$ at room temperature according to the manufacturer's protocol. Subsequently, the apoptosis of nalm-6 cells was analyzed by flow cytometry using a Guava easyCyte $8 \mathrm{HT}$ flow cytometer (EMD Millipore, Billerica, MA, USA) and Guava Incyte (version 3.1.1; EMD Millipore).

Western blot analysis. Nalm-6 cells were seeded at $4 \times 10^{5} / \mathrm{ml}$ in serum-free medium in a $25-\mathrm{cm}^{2}$ flask and treated with or without $1 \mathrm{U} / \mathrm{ml} \mathrm{BM-SCs-exosomes} \mathrm{in} \mathrm{the} \mathrm{presence}$ of $0.3 \mu \mathrm{g} / \mathrm{ml}$ VP16 for $48 \mathrm{~h}$. A group with nalm-6 cells alone served as control. Whole cell lysates were then extracted from cells suspended in radioimmunoprecipitation assay lysis buffer (catalog no., P0013B; Beyotime Institute of Biotechnology), which contained protease and phosphatase inhibitors. Lysates were separated by 12\% SDS-PAGE (marker ladder, 10-170 kDa; catalog no., SM0671/26616; Fermentas; Thermo Fisher Scientific, Inc.) and transferred to polyvinylidene difluoride membranes (EMD Millipore). The membranes were blocked in a blocking buffer of $20 \%$ skimmed-milk in TBS-Tween 20 for $1 \mathrm{~h}$ at room temperature, and incubated with primary antibody from rabbit against human $\beta$-actin (dilution, 1:1,000; catalog no., 20536-1-AP; ProteinTech Group, Inc., Wuhan, China), B-cell lymphoma 2 (BCL-2; dilution, 1:100; catalog no., ab7973; Abcam), BCL-2-like protein 4 (BAX; dilution, 1:2,000; catalog no., ab32503; Abcam), caspase-3 (dilution, 1:500; catalog no., 19677-1-AP; ProteinTech Group, Inc.), cleaved caspase-3 (dilution, 1:500; catalog no., 19677-1-AP; ProteinTech Group, Inc.), poly ADP-ribose polymerase (PARP; dilution, 1:400; catalog no., ab6079; Abcam), and cleaved PARP (dilution, 1:250; catalog no., ab6079; Abcam) overnight at $4^{\circ} \mathrm{C}$. The membranes were then exposed to goat anti-rabbit horseradish peroxidase-conjugated secondary antibody (dilution, 1:20,000; catalog no.,ZB-2301; OriGene Technologies, Inc., Beijing, China) for $1 \mathrm{~h}$ at room temperature. The bands were visualized and captured using ECL Western Blotting Substrate (EMD, Millipore) and C-Digit Image Studio (LI-COR, Nebraska, NE, USA) 47 software. 

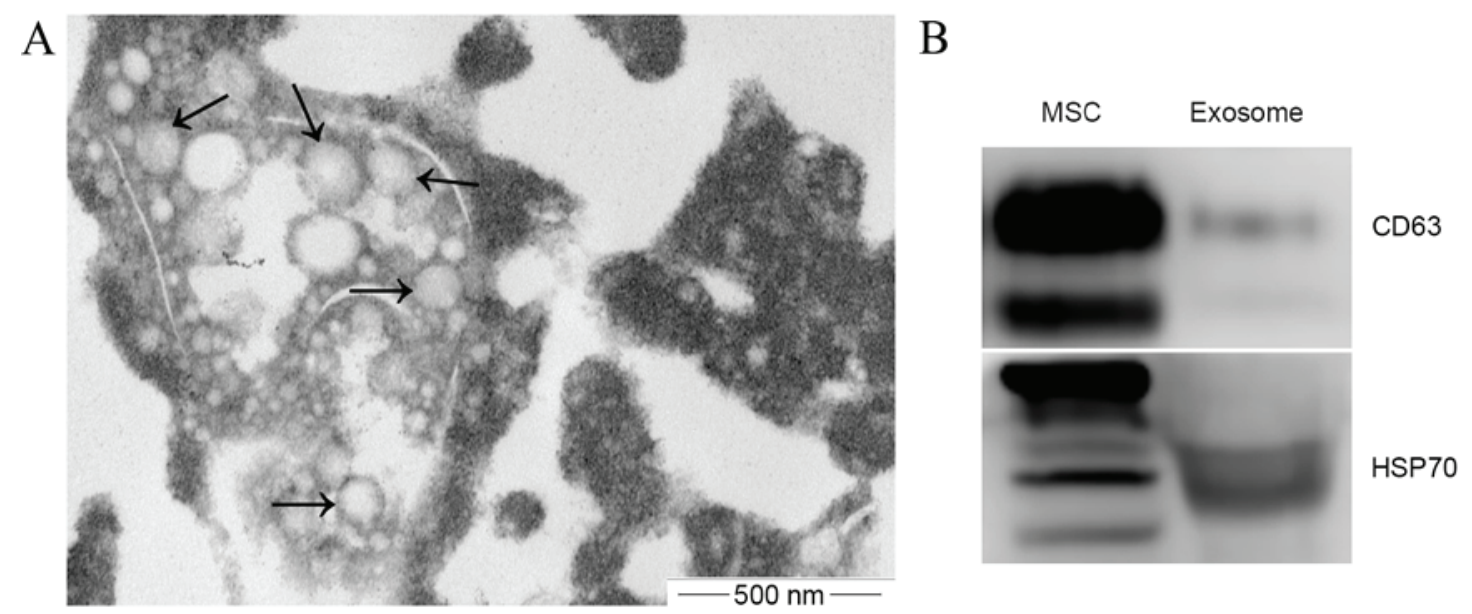

Figure 1. Characterization of BM-SC-exosomes. (A) Transmission electron microscopy images of exosomes derived from BM-SC cells. Scale bars represent $500 \mathrm{~nm}$, and arrows indicate typical exosomes. (B) Exosomal positive markers CD63 and HSP70 were detected in BM-SC-exosomes. BM-SC, bone marrow stromal cells; CD63, lysosomal-associated membrane protein 3; HSP70, 70 kilodalton heat shock proteins.

\section{Results}

Isolation and identification of BM-SCs-exosomes. Exosomes were successfully isolated from the conditioned medium of BM-SCs. The size and shape of the isolated exosomes were confirmed using transmission electron microscopy, and typical cup-shaped membrane particles with a diameter of 50-150 nm were observed (Fig. 1A). Western blot analysis showed that exosomal markers CD63 and HSP70 were detected in these exosomes (Fig. 1B). These results confirmed that the present study successfully isolated exosomes from the BM-SCs-conditioned medium.

BM-SCs-exosomes promote the viability of leukemia cells. BM-SCs are known to increase the viability of leukemia cells, but the role of exosomes in these actions is not clearly elucidated. In the present study, the effects of BM-SCs-derived exosomes on the cell viability of nalm- 6 cells were investigated in the presence or absence of VP16. As expected, the viability of nalm- 6 cells was increased subsequent to the cells being co-cultured with BM-SCs-derived exosomes in the presence or absence of VP16 compared with the untreated cells ( $\mathrm{P}=0.00081$ and $\mathrm{P}=0.00073$, respectively; Fig. $2 \mathrm{~A}$ and $\mathrm{B})$. These results demonstrated that BM-SCs-derived exosomes maintain the survival of nalm- 6 cells; thereby decreasing the latter's sensitivity to VP16.

BM-SCs-derived exosomes decrease the apoptosis of leukemia cells. To additionally explore the effects of exosomes on nalm- 6 cells, the present study evaluated the apoptosis of nalm- 6 cells subsequent to co-culturing with BM-SCs-derived exosomes. Fluorescence-activated cell sorting results showed that BM-SCs-derived exosomes decreased the apoptotic percentage of nalm- 6 cells (nalm- 6 and VP16 vs. nalm-6, VP16 and exosomes; $\mathrm{P}=0.00816$ early apoptosis and $\mathrm{P}=0.00415$ late apoptosis) induced by VP16 while increasing the percentage of live cells $(\mathrm{P}=0.00727)$ compared with the untreated cells (Fig. 3A and B). These data indicate that BM-SCs-derived exosomes protect nalm-6 cells from VP16-induced apoptosis to maintain its survival.
$B M$-SCs-derived exosomes regulate the apoptotic-associated protein levels in leukemia cells. In the present study, the expression levels of apoptotic-associated proteins BAX, BCL-2, caspase-3, cleaved caspase-3, PARP, and cleaved PARP were evaluated by western blot analysis. The results showed that the expression levels of BAX, cleaved caspase-3, and cleaved PARP were significantly upregulated in the VP16 group (BAX, $\mathrm{P}=0.00011$; cleaved caspase- $3, \mathrm{P}=0.00012$; cleaved PARP, $\mathrm{P}=0.00015$ ), but were low in the control group. However, their expressions were downregulated subsequent to treatment with exosomes for $48 \mathrm{~h}(\mathrm{BAX}, \mathrm{P}=0.00287$; cleaved caspase- $3, \mathrm{P}=0.00606$; cleaved PARP, $\mathrm{P}=0.00712$ ). The expression levels of BCL-2, caspase-3, and PARP were significantly downregulated in the VP16 group compared with those in the control group (BCL-2, $\mathrm{P}=0.00024$; caspase- $3, \mathrm{P}=0.00488$; PARP, $P=0.00083$ ), whereas they were significantly increased in the exosome-treated group compared with the VP16 group (BCL-2, $\mathrm{P}=0.00601$; caspase- $3, \mathrm{P}=0.00221$; $\mathrm{PARP}, \mathrm{P}=0.00671$ ) (Fig. 4A and B). These data indicate that apoptosis-associated proteins may be involved in the drug resistance of leukemia cells induced by BM-SCs-derived exosomes.

\section{Discussion}

Previous studies have reported that exosomes may mediate cell-to-cell communication $(12,13)$ and possess crucial roles in hematological malignancies. These studies have demonstrated the functions of exosomes in cell apoptosis (14), cell proliferation $(15,16)$, differentiation $(15)$, angiogenesis $(17,18)$, natural killer cell cytotoxicity (19) and induction of antitumor T-cell immunity (20). However, the role of exosomes in the pathogenesis and progression of leukemia has not yet been thoroughly evaluated. BM-SCs directly interact with leukemia cells and secrete soluble factors and other functional components to support the growth of these cells. However, the role of exosomes in these actions remains largely unclear. The present study provides novel evidence on the role of BM-SCs-derived exosomes in BM-SCs-induced leukemia cell growth, survival and drug resistance, leading to a better understanding of the interactions between 

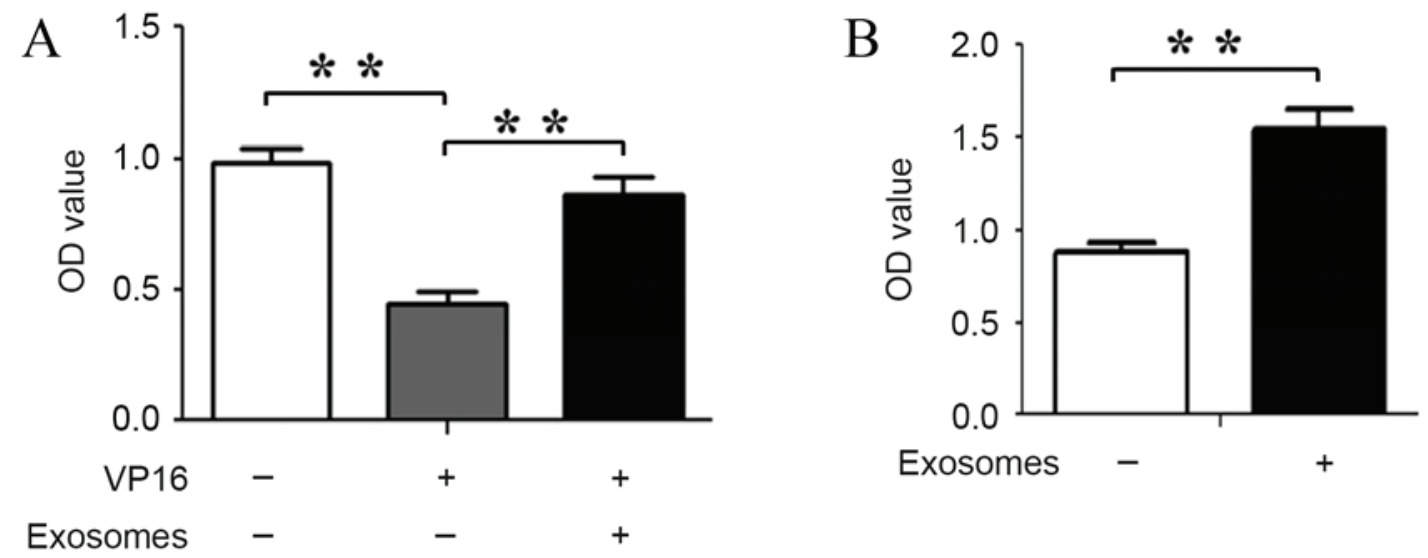

Figure 2. BM-SC-exosomes increase the viability of leukemic cells. (A) Exosomes derived from BM-SCs increase the viability of nalm- 6 cells in the presence of VP16. (B) In the absence of VP16, BM-SC-exosomes increase nalm- 6 cell viability. "P $<0.01$. BM-SC, bone marrow stromal cells; VP16, etoposide.
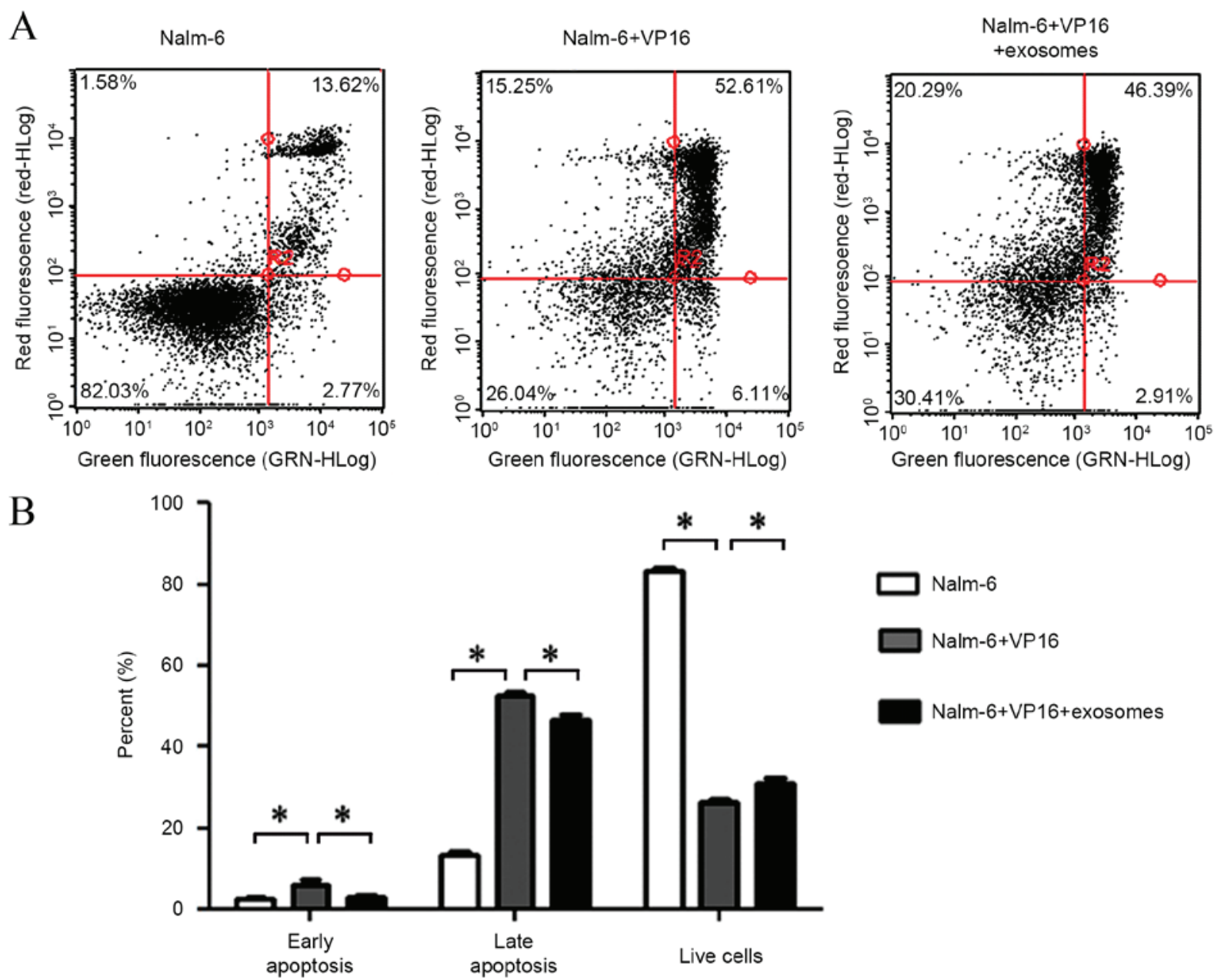

Figure 3. BM-SC-exosomes exhibited an increased capacity to resist VP16-induced apoptosis. (A) Effects of BM-SC-exosomes on nalm-6 cells in the presence of VP16. (B) Percentage of apoptotic and live nalm- 6 cells in the total cells. All values are expressed as the mean \pm standard deviation of three independent experiments. ${ }^{*} \mathrm{P}<0.05,{ }^{* *} \mathrm{P}<0.01$. BM-SC, bone marrow stromal cells; VP16, etoposide.

exosomes in the BM microenvironment and the leukemia cells. In the present study, exosomes were isolated by ultracentrifugation, and the exosomal morphology and markers were confirmed using electron microscopy and western blot analysis. The results of the present study demonstrated that exosomes are nanoparticles of $\sim 100 \mathrm{~nm}$ in size and exhibit a membrane-like bilayer with a typical cup-like shape. They commonly expressed CD63 and HSP70 markers.

BM-SCs affect the survival and proliferation of leukemia cells by activating anti-apoptotic and growth signaling pathways to secrete growth factors $(21,22)$. In the current study, the results demonstrate that BM-SCs-exosomes increased the 


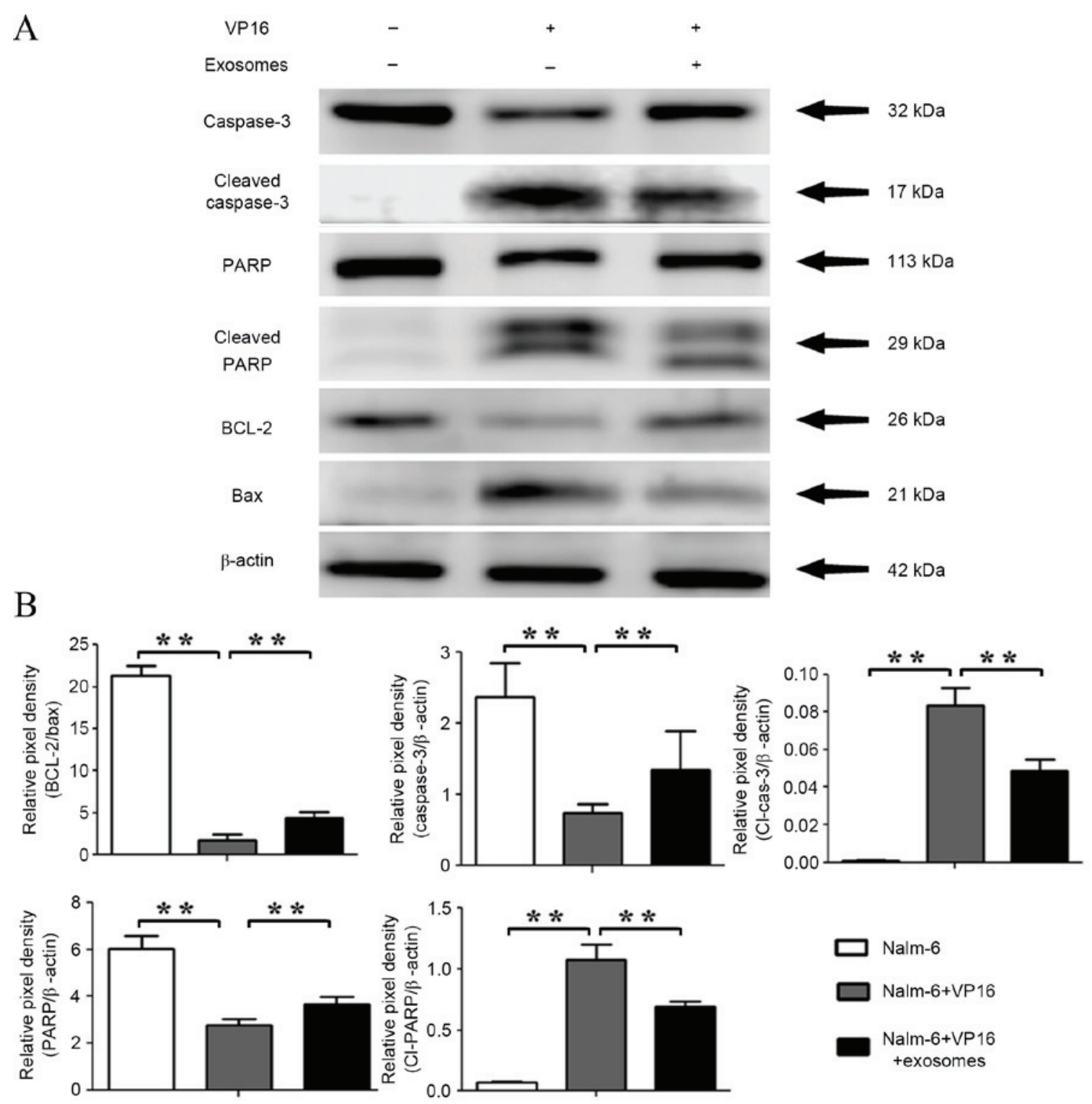

Figure 4. Effects of BM-SC-exosomes on the expression levels of apoptosis-associated proteins. (A) Nalm-6 cells in serum medium were treated with BM-SC-derived exosomes in the presence of $0.3 \mu \mathrm{g} / \mathrm{ml} \mathrm{VP16}$, and apoptosis-associated proteins BCL-2, BAX, caspase-3, and PARP were detected using western blot analysis. (B) Pixel densities of the proteins were quantified from 3 independent experiments and presented by histograms. The mean values \pm standard deviation for three independent experiments are shown. "P $<0.05,{ }^{* *} \mathrm{P}<0.01$. BM-SC, bone marrow stromal cells; VP16, etoposide; BCL-2, B-cell lymphoma 2; BAX, BCL-2-like protein 4; PARP, poly ADP-ribose polymerase; Cl-cas-3, cleaved caspase-3; Cl-PARP, cleaved PARP.

viability and survival of leukemia cells, and different pathways, such as BCL-2/BAX, caspase-3, and PARP are involved in these actions. BM-SCs-exosomes may maintain cell survival through the following ways: Stimulating target cells through surface-expressed receptors to induce signal transduction and activation of anti-apoptotic pathways in leukemic cells; horizontally transferring transcription factors and miRNAs to leukemic cells to activate relevant pathways; and transferring receptors to target cells to induce more signaling pathways (23). The current results show that $\mathrm{BM}-\mathrm{SC}$-exosomes blocked the reduction of protein expression levels of BCL-2, full-length caspase-3, and PARP induced by VP16; and inhibited the expression levels of BAX, cleaved caspase-3, and cleaved PARP. These results indicate that BM-SC-exosomes may protect nalm- 6 cells from VP16-induced cell apoptosis by regulating apoptosis-associated signal molecules to induce drug resistance.
Drug resistance is a major problem in clinical cancer treatment and a consequence of multiple factors, including inhibition of apoptosis, enhanced drug export, reduced drug uptake, enhanced DNA repair, and enhanced drug inactivation (24). Evasion of apoptosis is typical of numerous cancers and a frequent cause of therapeutic resistance.

Cell apoptosis is a type of programmed cell death that plays a critical role in normal tissue development and removal of damaged, old or infected cells. During the course of apoptosis, cells and nucleosomal DNA fragments undergo structural changes, including condensation of nucleus and cytoplasm and formation of small membrane-bound structures. In malignancies, the apoptosis program is inhibited in cancer cells, particularly in hematologic malignancies (25). Cell apoptosis is initiated by 2 signaling pathways, the intrinsic and extrinsic pathways. The intrinsic pathway is more commonly 
perturbed in lymphoid malignancies. The intrinsic pathway, also termed stress or mitochondrial pathway, is evolutionarily highly conserved and mainly controlled by the BCL-2 protein family (26). This protein family has two subgroups based on their pro-apoptotic or anti-apoptotic activity, namely, pro-apoptotic protein members [BAX, BCL-2 homologous antagonist killer (BAK), BCL-2-like protein 11 (BIM), BH3 interacting-domain death agonist, and BCL-2-associated death promoter] and pro-survival protein members (BCL-2, BCL-extra large, BCL like 2, myeloid cell leukemia 1, BCL-like protein, and BCL2 related protein A1) (27). Under normal conditions in healthy lymphoid cells, the pro-survival members of the BCL2 family constrain the essential cell death mediators BAX and BAK, thereby maintaining cell viability. Stress signals, such as DNA damage induced by chemotherapy, can trigger the activation of $\mathrm{BH} 3$-only proteins, such as BIM. These proteins can bind to and inactivate the pro-survival protein families, such as BCL-2, and allow the activation of pro-apoptotic protein families such as BAX and BAK. Once activated, BAX and BAK permeabilize the outer mitochondrial membrane and trigger the release of factors, such as cytochrome $\mathrm{c}$, which functions as a co-factor for the activation of caspases, including caspase- 3 , caspase- 8 and caspase-9; as well as damage the mitochondria which is the cell's major energy source (28). Caspases (cysteine-aspartic proteases) are proteolytic enzymes that play an important role in controlling cell death and inflammation. Caspase family members are classified into two subgroups, namely, upstream or initiator (caspase-1, -2, -4, -5, -8, -9, -10, -11 and 12) and downstream or effector (caspase-3, -6, -7 and -14). Caspase-3 plays an important role in cell apoptosis (29). PARP participates in DNA damage by attaching to DNA repair proteins. Adequate PARP expression is able to facilitate the DNA repair process; however, activation of PARP is also able to induce cell apoptosis (30). A recent study has shown that BM-SCs-exosomes can protect multiple myeloma cells from bortezomib-induced apoptosis to maintain their survival and induce drug resistance by blocking the significant reduction of BCL-2 and by reducing the expression levels of cleaved caspase-3 and PARP (31).

The present results show that VP16 can inhibit the expression of BCL-2 to allow the activation of BAX, which in turn activates the executioner caspase- 3 to increase the expression level of the cleaved form of PARP and induce the apoptosis of nalm- 6 cells. However, exosomes derived from BM-SCs significantly increased BCL-2, full-length caspase-3, and PARP, and decrease the protein levels of BAX, cleaved caspase-3, and cleaved PARP to reduce the apoptosis of nalm- 6 cells. These results indicate that BM-SCs-exosomes may be involved in the drug resistance of nalm- 6 cells to VP16 by regulating cell apoptosis. This provides new ideas and strategies for the clinical treatment of leukemia. However, additional research is required to determine the potential effects of these exosomes on the development, progression, and prognosis of leukemia.

\section{Acknowledgements}

Shandong Province Natural Science Foundation (grant nos., 2014GSF118131 and 201403010), The
Major State Basic Research Development Program (grant no., 2012CB966504), Shenzhen Science Development Fund (grant no., JCYJ20140418115449178), Basic Scientific Fund of Shandong University (grant no., 2014QLKY02\&2014QY003-11) and Science Foundation of Qilu Hospital of Shandong University (grant no., 2016QLQN23) all supported this study.

\section{References}

1. Wong RS and Cheong SK: Leukaemic stem cells: Drug resistance, metastasis and therapeutic implications. Malays J Pathol 34: 77-88, 2012.

2. Raetz EA and Bhatla T: Where do we stand in the treatment of relapsed acute lymphoblastic leukemia? Hematology Am Soc Hematol Educ Program 2012: 129-136, 2012.

3. Bianco P, Cao X, Frenette PS, Mao JJ, Robey PG, Simmons PJ and Wang CY: The meaning, the sense and the significance: Translating the science of mesenchymal stem cells into medicine. Nat Med 19: 35-42, 2013.

4. Meads MB, Hazlehurst LA and Dalton WS: The bone marrow microenvironment as a tumor sanctuary and contributor to drug resistance. Clin Cancer Res 14: 2519-2526, 2008.

5. Wong RY and Cheong SK: Role of mesenchymal stem cells in leukaemia: Dr. Jekyll or Mr. Hyde? Clin Exp Med 14: 235-248, 2014.

6. Mudry RE, Fortney JE, York T, Hall BM and Gibson LF: Stromal cells regulate survival of B-lineage leukemic cells during chemotherapy. Blood 96: 1926-1932, 2000.

7. Dias S, Choy M, Alitalo K and Rafii S: Vascular endothelial growth factor (VEGF)-C signaling through FLT-4 (VEGFR-3) mediates leukemic cell proliferation, survival, and resistance to chemotherapy. Blood 99: 2179-2184, 2002.

8. Benabbou N, Mirshahi P, Bordu C, Faussat AM, Tang R, Therwath A, Soria J, Marie JP and Mirshahi M: A subset of bone marrow stromal cells regulate ATP-binding cassette gene expression via insulin-like growth factor-I in a leukemia cell line. Int J Oncol 45: 1372-1380, 2014.

9. Record M, Subra C, Silvente-Poirot S and Poirot M: Exosomes as intercellular signalosomes and pharmacological effectors. Biochem Pharmacol 81: 1171-1182, 2011.

10. Vlassov AV, Magdaleno S, Setterquist R and Conrad R: Exosomes: Current knowledge of their composition, biological functions, and diagnostic and therapeutic potentials. Biochim Biophys Acta 1820: 940-948, 2012.

11. Lai RC, Arslan F, Lee MM, Sze NS, Choo A, Chen TS, Salto-Tellez M, Timmers L, Lee CN, El Oakley RM, et al: Exosome secreted by MSC reduces myocardial ischemia/reperfusion injury. Stem Cell Res 4: 214-222, 2010.

12. Huan J, Hornick NI, Shurtleff MJ, Skinner AM, Goloviznina NA, Roberts CT Jr and Kurre P: RNA trafficking by acute myelogenous leukemia exosomes. Cancer Res 73: 918-929, 2013.

13. Umezu T, Ohyashiki K, Kuroda M and Ohyashiki JH: Leukemia cell to endothelial cell communication via exosomal miRNAs. Oncogene 32: 2747-2755, 2013.

14. Raimondo S, Saieva L, Corrado C, Fontana S, Flugy A, Rizzo A, De Leo G and Alessandro R: Chronic myeloid leukemia-derived exosomes promote tumor growth through an autocrine mechanism. Cell Commun Signal 13: 8, 2015.

15. Ansa-Addo EA, Lange S, Stratton D, Antwi-Baffour S, Cestari I, Ramirez MI, McCrossan MV and Inal JM: Human plasma membrane-derived vesicles halt proliferation and induce differentiation of THP-1 acute monocytic leukemia cells. J Immunol 185: 5236-5246, 2010.

16. Roccaro AM, Sacco A, Maiso P, Azab AK, Tai YT, Reagan M, Azab F, Flores LM, Campigotto F, Weller E, et al: BM mesenchymal stromal cell-derived exosomes facilitate multiple myeloma progression. J Clin Invest 123: 1542-1555, 2013.

17. Mineo M, Garfield SH, Taverna S, Flugy A, De Leo G Alessandro R and Kohn EC: Exosomes released by K562 chronic myeloid leukemia cells promote angiogenesis in a Src-dependent fashion. Angiogenesis 15: 33-45, 2012 .

18. Tadokoro H, Umezu T, Ohyashiki K, Hirano T and Ohyashiki JH: Exosomes derived from hypoxic leukemia cells enhance tube formation in endothelial cells. J Biol Chem 288: 34343-34351, 2013. 
19. Reiners KS, Topolar D, Henke A, Simhadri VR, Kessler J, Sauer M, Bessler M, Hansen HP, Tawadros S, Herling M, et al: Soluble ligands for NK cell receptors promote evasion of chronic lymphocytic leukemia cells from NK cell anti-tumor activity. Blood 121: 3658-3665, 2013.

20. Chen W, Wang J, Shao C, Liu S, Yu Y, Wang Q and Cao X: Efficient induction of antitumor $\mathrm{T}$ cell immunity by exosomes derived from heat-shocked lymphoma cells. Eur J Immunol 36 1598-1607, 2006.

21. Fortney JE, Zhao W, Wenger SL and Gibson LF: Bone marrow stromal cells regulate caspase 3 activity in leukemic cells during chemotherapy. Leuk Res 25: 901-907, 2001.

22. Hall BM, Fortney JE, Taylor L, Wood H, Wang L, Adams S, Davis S and Gibson LF: Stromal cells expressing elevated VCAM-1 enhance survival of B lineage tumor cells. Cancer Lett 207: 229-239, 2004.

23. Camussi G, Deregibus MC, Bruno S, Grange C, Fonsato V and Tetta C: Exosome/microvesicle-mediated epigenetic reprogramming of cells. Am J Cancer Res 1: 98-110, 2011.

24. Fodale V, Pierobon M, Liotta L and Petricoin E: Mechanism of cell adaptation: When and how do cancer cells develop chemoresistance? Cancer J 17: 89-95, 2011.
25. Zaman S, Wang R and Gandhi V: Targeting the apoptosis pathway in hematologic malignancies. Leuk Lymphoma 55: 1980-1992, 2014

26. Delbridge AR and Strasser A: The BCL-2 protein family, BH3-mimetics and cancer therapy. Cell Death Differ 22: 1071-1080, 2015.

27. Reed JC: Bcl-2-family proteins and hematologic malignancies: History and future prospects. Blood 111: 3322-3330, 2008.

28. Green DR and Kroemer G: The pathophysiology of mitochondrial cell death. Science 305: 626-629, 2004.

29. Yi CH and Yuan J: The Jekyll and Hyde functions of caspases. Dev Cell 16: 21-34, 2009.

30. Bai P: Biology of poly(ADP-Ribose) polymerases: The factotums of cell maintenance. Mol Cell 58: 947-958, 2015.

31. Wang J, Hendrix A, Hernot S, Lemaire M, De Bruyne E, Van Valckenborgh E, Lahoutte T, De Wever O, Vanderkerken K and Menu E: Bone marrow stromal cell-derived exosomes as communicators in drug resistance in multiple myeloma cells. Blood 124: 555-566, 2014. 